PROCEEDINGS OF THE

AMERICAN MATHEMATICAL SOCIETY

Volume 129, Number 11, Pages 3417-3423

S 0002-9939(01)05938-X

Article electronically published on April 2, 2001

\title{
INCOMPRESSIBLE SURFACES IN LINK COMPLEMENTS
}

\author{
YING-QING WU
}

(Communicated by Ronald A. Fintushel)

\begin{abstract}
We generalize a theorem of Finkelstein and Moriah and show that if a link $L$ has a $2 n$-plat projection satisfying certain conditions, then its complement contains some closed essential surfaces. In most cases these surfaces remain essential after any totally nontrivial surgery on $L$.
\end{abstract}

A link $L$ in $S^{3}$ has a $2 n$-plat projection for some $n$, as shown in Figure 1, where a box on the $i$-th row and $j$-th column consists of 2 vertical strings with an $a_{i j}$ left-hand half twist; in other words, it is a rational tangle of slope $1 / a_{i j}$. See for example [BZ]. Let $n$ be the number of boxes in the even rows, so there are $n-1$ boxes in the odd rows. Let $m$ be the number of rows in the diagram. It was shown by Finkelstein and Moriah [FM1], [FM2] that if $n \geq 3, m \geq 5$, and if $\left|a_{i j}\right| \geq 3$ for all $i, j$, then the link exterior $E(L)=S^{3}-\operatorname{Int} N(L)$ contains some essential planar surfaces, which can be tubed on one side to obtain closed incompressible surfaces in $E(K)$. In this note we will prove a stronger version of this theorem, showing that $E(L)$ contains some essential surfaces if $n \geq 3$, the boxes at the two ends of the odd rows have $\left|a_{i j}\right| \geq 3$, and $a_{i j} \neq 0$ for the boxes which are not on the ends of the rows. We allow $a_{i j}=0$ for boxes at the ends of the even rows, and there is no restriction on $m$, the number of rows in the diagram. The argument here provides a much simpler proof to the above theorem of Finkelstein and Moriah. In FM2 that theorem was applied to show that if $L$ is a knot, then all surgeries on $L$ contain essential surfaces. Corollary 2 below generalizes this to the case when $L$ has multiple components, with a mild restriction that each component of $L$ intersects some "allowable" spheres.

We first give some definitions. Let $\alpha=\alpha\left(a_{1}, \ldots, a_{m}\right)$ be an arc running monotonically from the top to the bottom of the $2 n$-plat, such that $\alpha$ is disjoint from the boxes, and on the $i$-th row there are $a_{i}$ boxes on the left of $\alpha$. See Figure 1 for the arc $\alpha(1,1,1,2,2)$. The arc $\alpha$ is an allowable path if (i) each row has at least one box on each side of $\alpha$, and (ii) $\alpha$ intersects $L$ at $m+1$ points (so $\alpha$ intersects $L$ once when passing from one row to another). Note that the leftmost allowable path is $\alpha(1, \ldots, 1)$, which has on its left one box from each row.

Given an allowable path $\alpha=\alpha\left(a_{1}, \ldots, a_{m}\right)$, we can connect the two ends of $\alpha$ by an arc $\beta$ disjoint from the projection of $L$ to form a circle, then cap it off by two disks, one on each side of the projection plane, to get a sphere $S=S\left(a_{1}, \ldots, a_{m}\right)$,

Received by the editors February 22, 2000 and, in revised form, March 27, 2000.

1991 Mathematics Subject Classification. Primary 57N10, 57M25.

Key words and phrases. Incompressible surfaces, $2 n$-plat projections, Dehn surgery.

The author was supported in part by NSF grant \#DMS 9802558. 

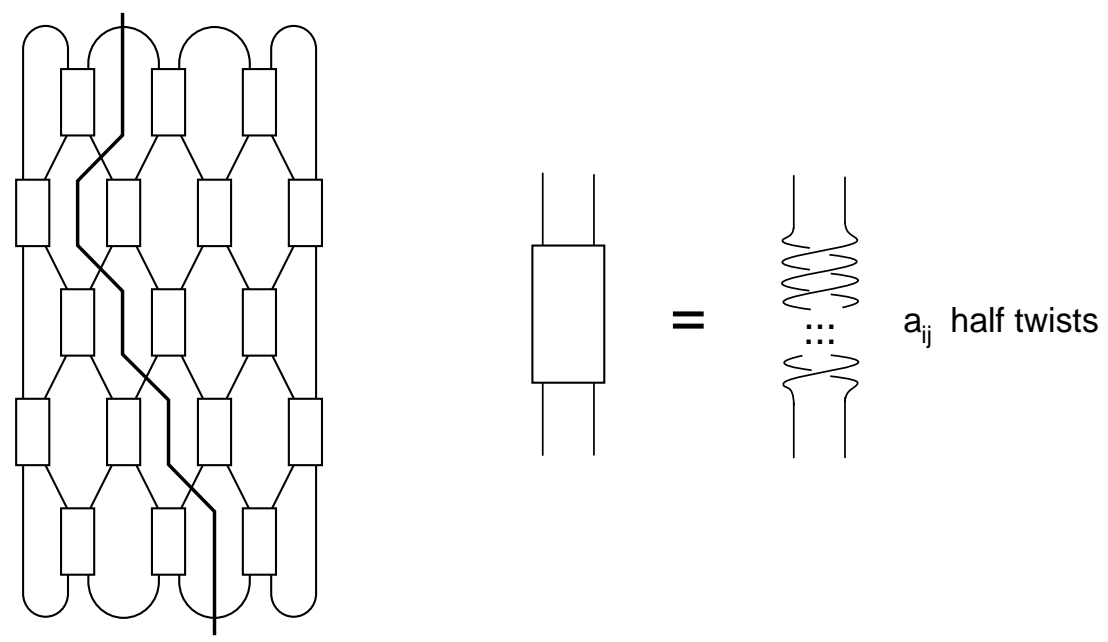

Figure 1.

called an allowable sphere. $S$ cuts $\left(S^{3}, L\right)$ into two tangles $(B, T)$ and $\left(B^{\prime}, T^{\prime}\right)$, where $(B, T)$ denotes the one on the left hand side of $S$. Let $P=P\left(a_{1}, \ldots, a_{m}\right)$ be the planar surface $S \cap E(L)$, which cuts $E(L)$ into two pieces $X=X\left(a_{1}, \ldots, a_{m}\right)$ and $X^{\prime}=X^{\prime}\left(a_{1}, \ldots, a_{m}\right)$, with $X=B \cap E(L)$ the one on the left of $P$. Let $F=F\left(a_{1}, \ldots, a_{m}\right)$ be the surface obtained by tubing $P$ on the left hand side; in other words, $F$ is the component of $\partial X$ containing $P$, pushed slightly into the interior of $E(L)$. Similarly, denote by $F^{\prime}=F^{\prime}\left(a_{1}, \ldots, a_{m}\right)$ the surface obtained by tubing $P$ on the right hand side.

Recall that a properly embedded surface $F$ in a 3-manifold $M$ is an essential surface if it is incompressible, $\partial$-incompressible, and is not boundary parallel. We define a surface $F$ on the boundary of $M$ to be essential if it is incompressible, $M \neq F \times I$, and there is no compressing disk of $\partial M$ which intersects $F$ at a single essential arc in $F$. Thus if $F$ is properly embedded in $M$, then it is essential if and only if after cutting along $F$ the two copies of $F$ are essential in the resulting manifold. A 3-manifold $M$ is $\partial$-irreducible if $\partial M$ is incompressible in $M$. Given a set $A$ in $M$, denote by $N(A)$ a regular neighborhood of $A$ in $M$.

Theorem 1. Suppose $L$ has a $2 n$-plat projection such that (i) $n \geq 3$; (ii) $a_{i j} \neq 0$ for $j \neq 0, n$; and (iii) $\left|a_{i j}\right| \geq 3$ for $i$ odd and $j=0$ or $n-1$. Let $S=S\left(a_{1}, \ldots, a_{m}\right)$ be an allowable sphere. Then $E(L)$ is irreducible, and the surfaces $F=F\left(a_{1}, \ldots, a_{m}\right)$ and $F^{\prime}=F^{\prime}\left(a_{1}, \ldots, a_{m}\right)$ are essential in $E(L)$.

Let $L=L_{1} \cup \ldots \cup L_{k}$ be a $k$ component link, let $r=\left(r_{1}, \ldots, r_{k}\right)$ be a set of slopes on $\partial N(L)$, with $r_{i}$ a slope on $\partial N\left(L_{i}\right)$. Then $L(r)$ denotes the $r$-Dehn surgery on $L$, which is the manifold obtained by gluing $k$ solid tori $V_{1}, \ldots, V_{k}$ to $E(L)$ so that each $r_{i}$ is identified with a meridian disk of $V_{i}$. The surgery and the slope $r$ are totally nontrivial if no $r_{i}$ is the meridian slope of $L_{i}$.

Corollary 2. Let $L$ be as in Theorem 1 . If each component of $L$ intersects some allowable sphere, then $L(r)$ is a Haken manifold for all totally nontrivial $r$, and the surfaces $F$ and $F^{\prime}$ in Theorem 1 remain incompressible in $L(r)$. 
Remark. (1) It is easy to see that $F=F\left(a_{1}, \ldots, a_{m}\right)$ being incompressible implies that $P=P\left(a_{1}, \ldots, a_{m}\right)$ is an essential planar surface in $E(L)$. With a similar proof to that of Theorem 1 one can show that $P$ is essential even if the condition $\left|a_{i j}\right| \geq 3$ in (iii) of Theorem 1 is replaced by $\left|a_{i j}\right| \geq 2$. This generalizes the main theorem of [FM1].

(2) When $n \leq 2$, the link is a 2-bridge link, so by [HT] $E(L)$ contains no closed essential surface. Hence the assumption $n \geq 3$ in Theorem 1 is necessary.

(3) By definition of $2 n$-plat projection, the number of rows $m$ is odd. If $m=1$ the link is a composite link, and our assumption implies that it is nonsplit. In this case $E(L)$ is irreducible, and the surfaces in the theorem are swallow-follow tori, which are essential. Therefore the theorem is true for $m=1$. We may thus assume that $m \geq 3$ in the proof of Theorem 1 .

(4) In Corollary 2, each component of $L$ intersects some allowable sphere if and only if no component of $L$ is on the left of $S(1, \ldots, 1)$ or on the right of $S(n-2, n-1, \ldots, n-2)$, which is equivalent to that $a_{i 1}$ and $a_{j, n-1}$ are odd for some odd $i, j$.

(5) The results remain true if we replace the twist tangles with rational tangles of slopes $p_{i j} / a_{i j}$ with $a_{i j}$ satisfying the conditions in the theorem, or certain kinds of more complicated tangles. However in this case the link diagram would not be in $2 n$-plat form.

A $p / q$ rational tangle is a pair $(B, T)$, where $B$ is a "pillow case" in $\mathbf{R}^{3}$ with corner points $(0, \pm 1, \pm 1)$, and $T$ is obtained by taking 2 arcs of slope $p / q$ on $\partial B$ connecting the four conner points of the pillow case, then pushing the interior of the arcs into the interior of $B$. The $x z$-plane intersects $\partial B$ in a circle $C$ of slope $\infty$, called a vertical circle on $\partial B$. Each component of $\partial B-C$ contains two points of $\partial T$. We need the following result about rational tangles.

Lemma 3. Suppose $(B, T)$ is a $p / q$ rational tangle, and $C$ a vertical circle on $\partial B$. Let $X=B-\operatorname{Int} N(T)$, and let $P$ be a component of $(\partial B \cap X)-C$.

(i) if $q \geq 1$, then $P$ is incompressible in $X$;

(ii) if $q \geq 2$, then $\partial X-C$ is incompressible in $X$;

(iii) if $q \geq 3$, then any compressing disk of $\partial X$ intersects $P$ at least twice.

Proof. (ii) Notice that when attaching a 2-handle to $X$ along the curve $C$, the manifold $X_{C}$ is the exterior of a 2-bridge link associated to the rational number $p / q$, which is nontrivial and nonsplit when $q \geq 2$. In particular, $\partial X_{C}$ is incompressible. If $D$ is a compressing disk of $\partial X$ disjoint from $C$, then since $X$ is a handlebody of genus 2, we can find a nonseparating compressing disk $D^{\prime}$ which is still disjoint from $C$. But then $D^{\prime}$ would remain a compressing disk in $X_{C}$, a contradiction.

(i) If $q \geq 2$ this follows from (ii) and the fact that $P$ is a subsurface of $\partial X-C$ whose complement contains no disk components. If $q=1, X$ is a product $P \times I$, and the result is obvious.

(iii) By (i) $P$ is incompressible, which also implies that $\partial X-P$ is incompressible because any simple loop on $\partial X-P$ is isotopic to one in $P$. By [Wu, Lemma 2.1] there is no compressing disk of $X$ intersecting $P$ at a single essential arc.

The following lemma is well-known. The proof is an easy innermost circle outermost arc argument, and will be omitted. 


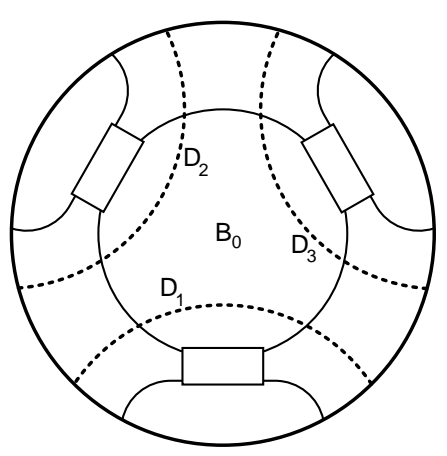

FiguRE 2.

Lemma 4. Let $F$ be an essential surface in a compact orientable 3-manifold $M$. If $M^{\prime}=M-\operatorname{Int} N(F)$ is irreducible, and no compressing disk of $\partial M^{\prime}$ is disjoint from the two copies of $F$ on $\partial M^{\prime}$, then $M$ is irreducible and $\partial$-irreducible.

We now proceed to prove Theorem 1. In the following, we will assume that $L$ is a link as in Theorem 1. By the remark above, we may assume $m \geq 3$.

Lemma 5. The manifold $X=X(1, \ldots, 1)$ is irreducible and $\partial$-irreducible.

Proof. Consider the tangle $(B, T)$ on the left of $S$. By an isotopy of $(B, T)$ we can untwist the boxes in $T$ which lie on the even rows of the projection of $L$, so the tangle $(B, T)$ is equivalent to the one shown in Figure 2, where each box corresponds to the first box on an odd row of the projection of $L$; hence there are $k=(m+1) / 2 \geq 2$ boxes $(k=3$ in Figure 2$)$. Let $D_{1}, \ldots, D_{k}$ be the disks represented by the dotted lines in Figure 2, which cuts $(B, T)$ into $k+1$ subtangles $\left(B_{0}, T_{0}\right), \ldots,\left(B_{k}, T_{k}\right)$, where $\left(B_{0}, T_{0}\right)$ is the one in the middle, which intersects all the $D_{i}$. Let $P_{i}=D_{i} \cap X$ be the twice punctured disk in $X$ corresponding to $D_{i}$. They cut $X$ into $X_{0}, \ldots, X_{k}$, with $X_{i}=B_{i}-\operatorname{Int} N\left(T_{i}\right)$ the tangle space of $\left(B_{i}, T_{i}\right)$.

We want to show that $\bigcup P_{i}$ is essential in $X$. Since each $\left(B_{i}, T_{i}\right), i \geq 1$, is a twist tangle with at least 3 twists, by Lemma 3 , the surface $P_{i}$ is essential in $X_{i}$. Now consider $X_{0}$. Put $Q=\partial B_{0}-\bigcup D_{i}$. If $D$ is a compressing disk of $Q$ in $X_{0}$, then it is a disk in $B_{0}$ disjoint from $T_{0} \cup\left(\cup D_{i}\right)$; but since $T_{0} \cup\left(\cup D_{i}\right)$ is connected, this would imply that one side of $D$ is disjoint from all $D_{i}$, hence $\partial D$ is a trivial curve on $Q$, which is a contradiction. Therefore $Q$ is incompressible in $X_{0}$. Assume there is a disk $D$ in $X_{0}$ such that $\partial D \cap\left(\bigcup P_{i}\right)$ has only one component. Since each string of $T_{0}$ has ends on different $D_{i}$, we see that $\partial D \cap \partial N\left(T_{0}\right)=\emptyset$, so $\partial D \cap\left(\bigcup P_{i}\right)$ is either a proper arc in some $D_{i}$ which separates the two points of $T_{0}$ on $D_{i}$, or it is a circle bounding a disk on $D_{i}$ containing exactly one point of $T_{0}$, or $\partial D$ can be isotoped into $Q$. The first two cases are impossible because then $D$ would be a disk in $B_{0}$ disjoint from $T_{0}$ and yet each component of $\partial B_{0}-\partial D$ contains an odd number of endpoints of $T_{0}$. The third case contradicts the incompressibility of $Q$. This completes the proof that $\bigcup P_{i}$ is an essential surface in $X$.

Notice that all $X_{i}$ are handlebodies, and hence irreducible. Since $Q$ is incompressible in $X_{0}$, and by Lemma 3 the surfaces $\partial X_{i}-P_{i} \subset \partial X_{i}-\partial D_{i}$ are incompressible in $X_{i}$ for $i \geq 1$, it follows from Lemma 4 that $X$ is irreducible and $\partial$-irreducible. 
Lemma 6. The manifold $X=X\left(a_{1}, \ldots, a_{m}\right)$ associated to an allowable sphere $S\left(a_{1}, \ldots, a_{m}\right)$ is irreducible and $\partial$-irreducible.

Proof. There is a sequence of allowable spheres $S_{1}, \ldots, S_{k+1}$, such that $S_{1}=$ $S(1, \ldots, 1), S_{k+1}=S\left(a_{1}, \ldots, a_{m}\right)$, and the noncommon part of $S_{i}, S_{i+1}$ bounds a single box in the projection of $L$, that is, $S_{i} \cup S_{i+1}-\operatorname{Int}\left(S_{i} \cap S_{i+1}\right)=\partial \widetilde{B}_{i}$ for some twist tangle $\left(\widetilde{B}_{i}, \widetilde{T}_{i}\right)$ with $a \neq 0$ left hand half-twists. Let $\left(B_{i}, T_{i}\right)$ be the tangle on the left of $S_{i}$, and let $X_{i}=B_{i}-\operatorname{Int} N\left(T_{i}\right)$. Similarly, let $\widetilde{X}_{i}=\widetilde{B}_{i}-\operatorname{Int} N\left(\widetilde{T}_{i}\right)$. Thus $X=X_{k+1}=X_{k} \cup_{P} \widetilde{X}_{k}$, where $P=X_{k} \cap \widetilde{X}_{k}$ is a twice punctured disk. By Lemma $5, X_{1}$ is irreducible and $\partial$-irreducible, and by induction on the length of the sequence we may assume that $X_{k}$ is irreducible and $\partial$-irreducible. Clearly $P$ is incompressible and $\partial$-incompressible on the $X_{k}$ side. If $|a| \geq 3$, then, by Lemma 3 , $P$ is also incompressible and $\partial$-incompressible on the $\widetilde{X}_{k}$ side, hence $P$ is an essential surface in $X$. Since $\partial \widetilde{X}_{k}-P$ is also incompressible in $\widetilde{X}_{k}$, and since $X_{k}$ and $\widetilde{X}_{k}$ are irreducible, it follows that $X=X_{k} \cup_{P} \widetilde{X}_{k}$ is irreducible and $\partial$-irreducible. Also, if $|a|=1$, then $\widetilde{X}_{k}$ is a product $P \times I$, so $X_{k+1} \cong X_{k}$, and the result follows.

It remains to prove the lemma for the case $|a|=2$. In this case there is a disk $D$ in $\widetilde{X}_{k}$ which intersects $P$ in a single $\operatorname{arc} \gamma$, cutting $\left(\widetilde{X}_{k}, P\right)$ into a pair $(A \times I, A \times \partial I)$, where $A$ is an annulus. Thus

$$
X=X_{k} \cup_{P} \widetilde{X}_{k}=\left(X_{k} \cup_{\gamma \times I}(D \times I)\right) \cup_{A \times \partial I}(A \times I) \cong X_{k} \cup_{A \times \partial I}(A \times I) .
$$

Since a compressing disk of $\partial(A \times I)$ intersects $A \times \partial I$ at least twice, by the same argument as above, one can show that $A \times \partial I$ is essential in $X$, and $X$ is irreducible and $\partial$-irreducible.

Proof of Theorem 1. Let $F, F^{\prime}$ be the surfaces in the theorem, isotoped slightly to be disjoint from each other. Then $F \cup F^{\prime}$ cuts $E(L)$ into three parts: The component on the left of $F$ is homeomorphic to $X$, the one on the right of $F^{\prime}$ is homeomorphic to $X^{\prime}$, and the one $X^{\prime \prime}$ between $F$ and $F^{\prime}$ is the union of $P \times I$ and $Q \times I$, where $Q$ is the set of tori in $\partial E(L)$ which intersect $\partial P$. We have shown in Lemma 6 that $X$ is irreducible and $\partial$-irreducible, and because of symmetry, so is $X^{\prime}$. Now $X^{\prime \prime}$ can be cut into $F \times I$ along some (essential) meridional annuli in $Q \times I$, hence by Lemma 4 it is irreducible and $\partial$-irreducible. Since $F$ and $F^{\prime}$ have genus at least 2 , they are not boundary parallel. It follows that $F \cup F^{\prime}$ is essential in $X$, and $X$ is irreducible.

Proof of Corollary 2. Let $S_{1}, \ldots, S_{k}$ be a set of disjoint allowable spheres, so that $S_{1}=S(1, \ldots, 1), S_{k}=S(n-2, n-1, \ldots, n-2)$, and there is only one box of the projection of $L$ between $S_{i}$ and $S_{i+1}$. These spheres are similar to those in the proof of Lemma 6 , except that they are now mutually disjoint, so the manifold between $S_{i}$ and $S_{i+1}$ is a product $S^{2} \times I$.

Let $F_{i}$ be the essential surfaces corresponding to $S_{i}$, as defined before Theorem 1 , isotoped slightly so that they are disjoint from each other. Also, isotope $F_{k}^{\prime}$ to be disjoint from $F_{k}$. Then the set of $k+1$ surfaces $F_{1}, F_{2}, \ldots, F_{k}, F_{k}^{\prime}$ cuts $E(L)$ into $k+2$ components $Y_{0}, \ldots, Y_{k+1}$, where $Y_{0}$ is the manifold $X(1, \ldots, 1)$ on the left of $F_{1}, Y_{k+1}=X^{\prime}(n-2, n-1, \ldots, n-2)$ is the manifold on the right of $F_{k+1}$, $Y_{k}$ is between $F_{k}$ and $F_{k}^{\prime}$, and for $1 \leq i \leq k-1, Y_{i}$ is between $F_{i}$ and $F_{i+1}$. Since all the $F_{i}$ and $F_{k}^{\prime}$ are essential, we see that $Y_{i}$ are all irreducible and $\partial$-irreducible. We need to show that the manifold $\widehat{Y}_{i}$ obtained from $Y_{i}$ by Dehn filling on its toroidal boundary components (if any), with slopes the corresponding subset of $r$, 


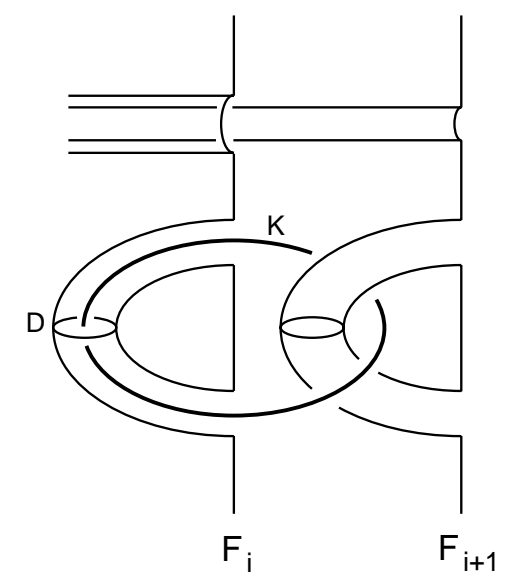

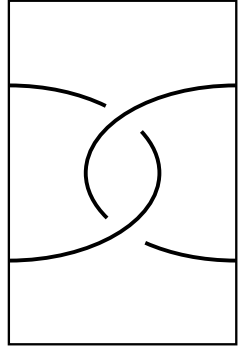

$\left(B_{i}^{\prime}, T_{i}^{\prime}\right)$

FIgURE 3.

is still irreducible and $\partial$-irreducible. The result will then follow by gluing the pieces together along $F_{i}$ and $F_{k}^{\prime}$.

Our assumption implies that $Y_{0}$ and $Y_{k+1}$ are disjoint from $\partial E(L)$, hence $\widehat{Y}_{i}=Y_{i}$ for $i=0, k+1$. Now $Y_{k}$ is a regular neighborhood of $P \cup Q$, where $P=S_{k} \cap$ $E(L)$, and $Q$ is the set of tori in $\partial E(L)$ which intersect $P$. Since $S$ is separating, each component $Q_{j}$ of $Q$ intersects $\partial P$ at least twice, so there are two nonparallel essential annuli in $Y_{k}$, each having a boundary component on $Q_{j}$ with meridional slope. Applying Menasco's theorem [Me] and Scharlemann's theorem [Sch] on each component of $Q$, we see that after any totally nontrivial Dehn filling on $Q$ the manifold $\widehat{Y}_{k}$ is still irreducible and $\partial$-irreducible.

Now assume $1 \leq i \leq k-1$. Let $\left(B_{i}^{\prime}, T_{i}^{\prime}\right)$ be the twist tangle between $S_{i}$ and $S_{i+1}$. Notice that if the twist number $a$ of $T_{i}^{\prime}$ is odd, then $Y_{i}$ contains no component of $\partial E(L)$, so $\widehat{Y}_{i}=Y_{i}$ and we are done. If $a$ is even, then the tangle $\left(B_{i+1}, T_{i+1}\right)$ on the left of $S_{i+1}$ may contain a loop $K$ intersecting the twist tangle $\left(B_{i}^{\prime}, T_{i}^{\prime}\right)$, so $Y_{i}$ may contain a single component $Q$ of $\partial E(L)$.

Let $Y_{i}(m)$ be the manifold obtained by the trivial Dehn filling on $Q$. Then $F_{i}$ has a compressing disk $D$ in $Y_{i}(m)$ intersecting the core $K$ of the Dehn filling solid torus only once, so $K$ is not a cable knot in $Y_{i}(m)$. See Figure 3. It follows from Sch that after surgery the manifold $\widehat{Y}_{i}$ is irreducible. Also, by [CGLS, Theorem 2.4.3] $\widehat{Y}_{i}$ is $\partial$-irreducible if the surgery slope $r_{j}$ on the torus $Q$ intersects the meridian slope $m$ at least twice. Now if $r_{j}$ intersects $m$ only once, then $m$ is a longitude after the surgery, hence the manifold $\widehat{Y}_{i}$ is homeomorphic to the one obtained by cutting $Y_{i}$ along the annulus $D \cap Y_{i}$, denoted by $\widetilde{Y}_{i}$. Now there is an annulus $A$ in $B_{i+1}-\operatorname{Int} B_{i}$ ( $B_{i}$ is the ball on the left of $S_{i}$ ) separating the twist tangle $\left(B_{i}^{\prime}, T_{i}^{\prime}\right)$ from the other $\operatorname{arcs}$ of $L$, which cuts $\widetilde{Y}_{i}$ into $\widetilde{X} \cong B_{i}^{\prime}-\operatorname{Int} N\left(T_{i}^{\prime}\right)$ and some $G \times I$, where $G$ is a subsurface of $F_{i}$ with one boundary component. Clearly $A$ is essential in $G \times I$. Since the twist number $a$ is even, our assumption in Theorem 1 implies that $|a| \geq 2$. Hence by Lemma 3 the surface $\partial \widetilde{X}-A$ is incompressible in $\widetilde{X}$, which implies that $A$ is essential in $\widetilde{X}$. It follows that $\widetilde{Y}_{i}$ is irreducible and $\partial$-irreducible. 


\section{REFERENCES}

[BZ] G. Burde and H. Zieschang, Knots, de Gruyter Studies in Math. 5, 1985. MR 87b:57004

[CGLS] M. Culler, C. Gordon, J. Luecke and P. Shalen, Dehn surgery on knots, Annals Math. 125 (1987), 237-300. MR 88a:57026, correction MR 89c:57015

[FM1] E. Finkelstein and Y. Moriah, Closed incompressible surfaces in knot complements, Trans. Amer. Math. Soc. 352 (2000), 655-677. MR 2000c:57007.

[FM2] - Tubed incompressible surfaces in knot and link complements, Topology Appl. 96 (1999), 153-170. CMP 99:16

[HT] A. Hatcher and W. Thurston, Incompressible surfaces in 2-bridge knot complements, Inv. Math. 79 (1985), 225-246. MR 86g:57003

[Me] W. Menasco, Closed incompressible surfaces in alternating knot and link complements, Topology 23 (1984), 37-44. MR 86b:57004

[Sch] M. Scharlemann, Producing reducible 3-manifolds by surgery on a knot, Topology 29 (1990), 481-500. MR 91i:57003

[Wu] Y-Q. Wu, The classification of nonsimple algebraic tangles, Math. Ann. 304 (1996), 457-480. MR 97b:57010

Department of Mathematics, University of Iowa, Iowa City, Iowa 52242

E-mail address: wu@math.uiowa.edu 